\title{
The Effect of Superoxide Dismutase Enzyme Inhibition on Renal Microcirculation of Spontaneously Hypertensive-Stroke Prone and Wistar Rats
}

\author{
A. F. AHMEDA ${ }^{1}$, M. G. RAE ${ }^{2}$, L. M. ANWEIGI ${ }^{3}$, M. F. AL OTAIBI ${ }^{1}$, A. A. AL-MASRI ${ }^{1}$, \\ E. J. JOHNS ${ }^{2}$
}

${ }^{1}$ Department of Physiology, College of Medicine, King Saud University, Riyadh, Kingdom of Saudi Arabia, ${ }^{2}$ Department of Physiology, University College Cork, Cork, Republic of Ireland, ${ }^{3}$ College of Dentistry, Princess Nourah Bint Abdulrahman University, Riyadh, Kingdom of Saudi Arabia

Received April 18, 2017

Accepted February 9, 2018

On-line May 10, 2018

\section{Summary}

A significant factor in the development of hypertension may be excessive vasoconstriction within the renal medulla. This study therefore investigated the role of superoxide dismutase (SOD) in the regulation of renal medullary and cortical blood perfusion (MBP and CBP, respectively) in both stroke-prone spontaneously hypertensive rats (SHRSP) and normotensive Wistar rats. CBP and MBP were measured before and after intra-renal infusion of the SOD inhibitor, diethyldithio-carbamic acid (DETC). Under basal conditions, mean arterial pressure was significantly greater in SHRSP than Wistar rats, but both MBP and heart rate $(H R)$ were significantly lower in SHRSP relative to Wistar rats $(P<0.05, \mathrm{n}=7$ in both groups). Infusion of DETC $(2 \mathrm{mg} / \mathrm{kg} / \mathrm{min})$ into the cortico-medullary border area of the kidney significantly decreased MBP in the SHRSPS (by $28 \pm 3 \%, n=7, P<0.05$ ), indicating a greater vasoconstriction within this vascular bed. However, DETC also significantly decreased MBP in Wistar rats to a similar extent $(24 \pm 4 \%, n=7, P<0.05)$. These results suggest that superoxide anions play a significant role in reducing renal vascular compliance within the renal medulla in both normotensive and hypertensive animals, although the responses are not greater in the hypertensive relative to the control animals.

\section{Key words}

Medullary blood perfusion - DETC • Reactive oxygen species • SHRSP • Renal microcirculation

\section{Corresponding author}

A. F. Ahmeda, Department of Physiology, College of Medicine, King Saud University, Riyadh, Kingdom of Saudi Arabia. Fax: +966114671040. E-mail: aahmeda@ksu.edu.sa

\section{Introduction}

The kidney has a very specialised blood supply and, despite receiving a proportionally large component of cardiac output, is very sensitive such that damage will occur if this blood supply is impaired either acutely or chronically. Although reactive oxygen species (ROS) are by-products of normal metabolic processes within cells, their build up within vascular smooth muscles can lead to oxidative stress, with the primary defence against such a build-up provided by SOD, which is able to scavenge superoxide anions (Halliwell and Gutteridge 1986).

The spontaneously hypertensive rat (SHR) has been widely used as a model of human essential hypertension as it develops complications associated with hypertension, such as cerebral and myocardial lesions, which are similar to those occurring in humans (Ahmeda and Alzoghaibi 2016, Okamoto and Aoki 1963). SHRs have chronically elevated blood pressure (BP) and increased vascular resistance relative to normotensive controls (Nagaoka et al. 1981). This is due, in part, to structural changes that occur within the cardiovascular system such as hypertrophy, impaired vascular endothelium-dependent vasodilatation and vascular 
remodelling caused by extracellular matrix deposition (Park et al. 2002). All of these factors combine to provoke increased peripheral resistance which, in turn, contributes to an increase in mean arterial pressure.

A substrain of SHR, the stroke-prone spontaneously hypertensive rat (SHRSP), exhibits a more severe hypertensive phenotype than the classical SHR and, indeed, displays a very high risk of stroke by 9-13 months of age (Tagami et al. 1987, Okamoto et al. 1974). Further, in addition to the increased renal vascular resistance mentioned above, SHRSPs display a greater reactivity to vasoactive substances such as noradrenaline and angiotensin II (Berecek et al. 1980) compared to normotensive rats, which further exacerbates their hypertensive state.

As acute elevations in arterial BP markedly increase arteriolar superoxide anion production, it is thought that this may in turn lead to an impairment of endothelial function, a result of which may be to set the stage for increased reactivity to vasoconstrictor stimuli and the development of hypertension (Harrison 1997, Suzuki et al. 1995).

Vascular differences between the SHRSP and Wistar rats, such as increased vascular resistance, may be due to an alteration in ROS and nitric oxide (NO) availability. For example, in SHRs, superoxide anion concentration is significantly elevated within the renal medulla compared to normotensive rats. Furthermore, Kerr et al. (1999) also confirmed that NO production was greater in SHRs compared with normotensive rats as a possible compensatory mechanism for the increased ROS production. Therefore, with regard to the greater hypertension observed within SHRSPs it may be that superoxide anion production overrides any enhanced NO generation and thereby counteracts its vasodilatory effects.

There is persuasive body of evidence that the renal medulla may be involved in the development of hypertension (Ahmeda and Alzoghaibi 2016, Mori et al. 2012, Cowley et al. 1995).

In this study, we investigated the effects of pharmacologically modifying the state of oxidative stress in the kidney of SHRSPs with a SOD inhibitor, and the consequences of blocking the SOD enzyme with DETC on medullary and cortical blood perfusion of SHRSPs. The findings in SHRSPs were compared to those recorded in normotensive Wistar rats.

\section{Methods}

All procedures were performed in accordance with European Community Directive 2010/63/EU and were approved by University College Cork Animal Experimentation Ethics Committee.

Four groups of male Wistar and SHRSP rats (7 rats in each experimental group) with body weight ranging from 250 to $350 \mathrm{~g}$ ( 12 weeks old), were housed in the Biological Services Unit at University College Cork for at least one week prior to use. The animals were given free access to standard chow and water until $12 \mathrm{~h}$ before surgery when food was restricted. Anaesthesia was induced with a $1 \mathrm{ml}$ bolus dose i.p. of chloralose/ urethane, 16.5 and $250 \mathrm{mg} / \mathrm{ml}$, respectively, and was maintained using supplemental doses of $0.05 \mathrm{ml}$ given i.v. every $30 \mathrm{~min}$.

The trachea was cannulated to assist respiration by providing a patent airway as well as facilitating the removal of any secretions as necessary.

The right femoral vein was cannulated for the infusion of saline and supplemental doses of anaesthetic, whereas the right femoral artery was cannulated for the measurement of blood pressure and heart rate. The left kidney was exposed through a flank incision and supported in a cup holder to eliminate respiratory movement. A small cannula was then inserted $4.5 \mathrm{~mm}$ into the cortico-medullary border (CMB) area of the kidney for infusion of saline or drugs at $1.0 \mathrm{ml} / \mathrm{h}$ as described previously (Ahmeda and Johns 2012, Ahmeda et al. 2013, Ahmeda et al. 2017).

Two laser-Doppler microprobes (each $0.5 \mathrm{~mm}$ diameter) were inserted 1.5 and $4.0 \mathrm{~mm}$ into the kidney to measure cortical and medullary perfusion, respectively. Following surgery, animals were allowed to stabilise for 90 min prior to experimentation.

Upon completion of each experiment, animals were euthanised by anaesthetic overdose and the kidneys were sectioned to confirm the location of the flow probes.

\section{Drug administration}

After a post-surgery stabilisation period of $90 \mathrm{~min}$, each group of animals received one of two treatments described below.

\section{Control groups}

The control groups ( $\mathrm{n}=7$, for both Wistar and SHRSP) received the vehicle, which was the solution $(0.9 \% \mathrm{NaCl})$ in which the drugs were dissolved. This solution was infused into the CMB area of the kidney. 


\section{DETC groups}

This groups $(\mathrm{n}=7$, for each group) received diethyldithio-carbamic acid (DETC), a SOD inhibitor (at a rate of $2 \mathrm{mg} / \mathrm{kg} / \mathrm{min}$ ). DETC has a short half-life in vivo of about 5 to $20 \mathrm{~min}$ (Voll et al. 1999). The dose of DETC chosen for the present study produces a physiological effect on renal microvasculature as approved in our lab previously (Ahmeda and Johns 2012). The compound was dissolved in normal saline and then infused into the CMB area of the kidney.

\section{Experimental protocol}

Baseline readings for cortical and medullary perfusion (CBP, MBP), mean arterial pressure (MAP) and heart rate (HR) were obtained over the $16 \mathrm{~min}$ period prior to the start of the renal interstitial infusion. Vehicle (saline) or DETC $(2 \mathrm{mg} / \mathrm{kg} / \mathrm{min})$, were infused for $30 \mathrm{~min}$, after which a further set of readings were taken over 16 min while the infusion continued.

\section{Statistical analysis}

Data are presented as mean \pm standard error of the mean (SEM). The SEM was used as a measure of data dispersion. The statistical significance of any druginduced changes in the measured parameters was evaluated using Student's paired t-test within the groups. For inter-group comparisons, two-way analysis of variance (ANOVA), followed by Tukey's test, was used. Effects were considered significant when $P<0.05$.

\section{Results}

Effect of intra-renal infusion of vehicle on $C B P$ and $M B P$ in SHRSP vs. Wistar rats

Initial experiments sought to determine the effect(s) of a $1 \mathrm{ml} / \mathrm{h}$ infusion of saline into the $\mathrm{CMB}$ on baseline renal regional blood flow. Average baseline levels of MAP, HR, CBP, and MBP for all experiments are summarised in Table 1.

As can be seen in Table 1, MAP in the SHRSPs was significantly elevated relative to that in the Wistar rats before infusing the vehicle $(134 \pm 4 \mathrm{~mm} \mathrm{Hg}$ in SHRSPs vs. $105 \pm 4 \mathrm{~mm} \mathrm{Hg}$ in Wistar rats, $\mathrm{n}=7 ; P<0.05$ ). However, it is interesting to note that MBP in SHRSPs was significantly lower in comparison to that in the Wistar rats $(53 \pm 3 \mathrm{PU}$ in SHRSP vs. $79 \pm 7 \mathrm{PU}$ in Wistar rats, $\mathrm{n}=7 ; P<0.05)$.

The CBP of SHRSP rats was close to the average CBP values in Wistar rats and was also relatively stable over the period of intramedullary infusion of vehicle.

No significant changes in any of the baseline parameters mentioned in Table 1 were found over the period of time after the vehicle was infused in either the normotensive or hypertensive groups of animals (the data not shown). It is also notable that HR was significantly reduced in the SHRSPs relative to Wistar rats.

Table 1. Shows baseline values of body weight MAP, CBP, MBP and HR.

\begin{tabular}{lcccc}
\hline \multirow{2}{*}{ Parameter } & \multicolumn{2}{c}{ Wistar } & \multicolumn{2}{c}{ SHRSP } \\
\cline { 2 - 5 } & Vehicle & DETC & Vehicle & DETC \\
\hline$n$ & 7 & 7 & 7 & 7 \\
Weight & $301 \pm 12$ & $296 \pm 18$ & $321 \pm 16$ & $318 \pm 14$ \\
Baseline MAP (mm Hg) & $105 \pm 4$ & $100 \pm 3$ & $134 \pm 4^{*}$ & $123 \pm 4^{*}$ \\
Baseline CBP (PU) & $165 \pm 19$ & $196 \pm 17$ & $155 \pm 7$ & $148 \pm 17$ \\
Baseline MBP (PU) & $79 \pm 7$ & $109 \pm 9$ & $53 \pm 3^{*}$ & $74 \pm 8^{*}$ \\
Baseline HR (beats/min) & $386 \pm 14$ & $403 \pm 16$ & $270 \pm 20^{*}$ & $254 \pm 13^{*}$ \\
\hline
\end{tabular}

Values were obtained from vehicle and DETC treated groups, prior to any infusion, of SHRSPs and Wistar rats. Statistical analysis was performed using a two-way ANOVA test. * indicates $P<0.05$, when baseline values of SHRSP were compared with those of Wistar rats.

Effect of renal medullary interstitial infusion of DETC on CBP and MBP in SHRSP vs. Wistar rats

Intra-medullary infusion of the SOD inhibitor, DETC ( $2 \mathrm{mg} / \mathrm{kg} / \mathrm{min})$, into the CMB had no significant effect on MAP or HR in either the SHRSP or Wistar rats. DETC did lower CBP in both SHRSP and Wistar rats but this was not statistically significant (Fig. 1). However, DETC did significantly decrease MBP in both SHRSP and Wistar rats, by $28 \pm 3 \%$ and $24 \pm 4 \%$, respectively $(P<0.05, \mathrm{n}=7$ in both groups), with its inhibitory effect on MBP in the SHRSPs being slightly greater than that seen in Wistar rats, albeit not so significantly (Fig. 1). 


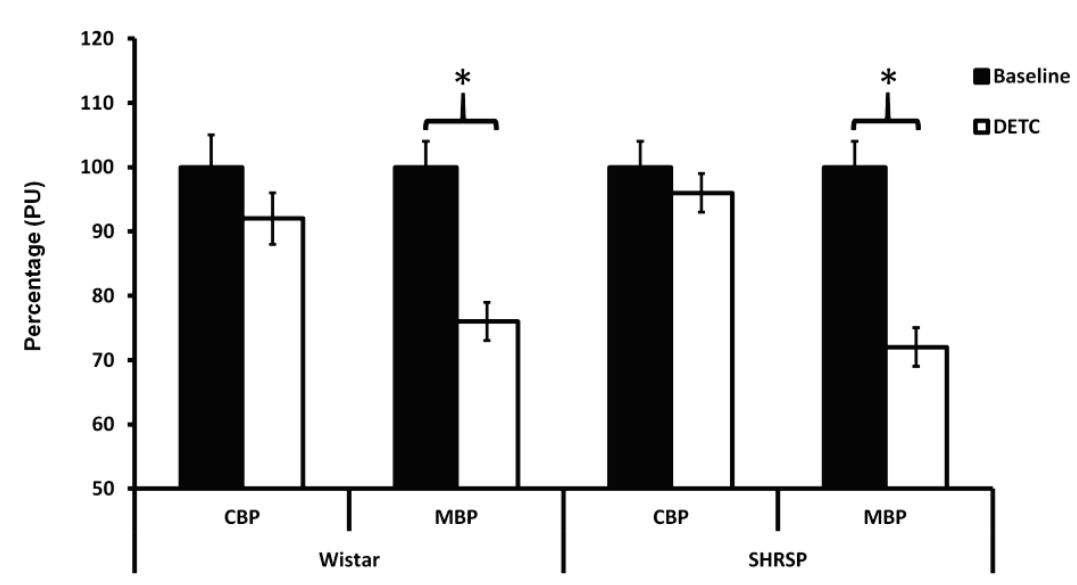

Fig. 1. The effect of DETC infusion into the CMB area on CBP and MBP in both SHRSPs and Wistar rats. Within group, and between group, data analysis was performed using Student's paired t-test and two-way ANOVA, respectively. * Indicates significant difference between the DETC treated group and the respective baseline $(P<0.05)$.

\section{Discussion}

\section{Control study}

It was evident from the control group of animals that MBP was lower than CBP in both SHRSP and Wistar rat strains, which is consistent with the observations from our previous studies on SHRSP and Wistar rats (Ahmeda and Johns 2012, Ahmeda et al. 2013, Ahmeda et al. 2017). Our findings are also in line with those reported by other studies using SpragueDawley rats (Chen et al. 2003, Zou et al. 2001). Notably however, medullary perfusion was further reduced (significantly) in SHRSPs relative to that recorded in the Wistar control rats, which indicates increased renal vascular resistance in these animals relative to controls.

The aim of the second part of the study was to investigate how increasing the level of oxidative stress within the kidney (by pharmacologically evoking an increase in the basal concentration of superoxide anions) affected cortical and medullary blood perfusion in both normotensive and hypertensive rats. The results of these experiments are discussed below.

\section{DETC study}

This study was undertaken in order to determine the contribution, if any, of oxidative stress to the control of renal haemodynamics by infusing the SOD inhibitor, DETC, locally into the renal CMB area.

Using this protocol we demonstrated that DETC caused a significant decrease in MBP in both anaesthetised Wistar and SHRSPs. This effect was presumably due to the exaggerated oxidative stress initiated by the DETC-induced increase in superoxide anion concentration within the renal medulla. Thus, as superoxide anions are potent vasoconstrictors of renal vasculature (Staiculescu et al. 2014, Moss et al. 2013), their increased production provoked a reduction in blood perfusion within the kidney.

These findings are in agreement with a previous study by Zou et al. (2001) who demonstrated that intra-medullary administration of DETC at a rate of $0.5 \mathrm{mg} / \mathrm{kg} / \mathrm{min}$ evoked vasoconstriction and anti-natriuretic effects and that DETC had a significantly greater action on MBP when compared with CBP. Nonetheless, it should be noted that this particular study was conducted on anaesthetised Sprague-Dawley rats only (Zou et al. 2001).

It is clear that in the present study the reduction in perfusion was restricted to the renal medulla, i.e. DETC infusion did not cause any significant changes in either systemic arterial BP or to CBP in either SHRSPs or Wistar rats. This finding is in agreement with a previous report which demonstrated that the enhancement of superoxide activity induced by DETC occurred mostly in the medulla in anaesthetised dogs (Majid and Nishiyama 2002), although they also observed an effect on the renal cortex, which might have been due to the different methods of drug administration.

It is generally accepted that superoxide-induced vasoconstriction is mainly caused by abolition of NO-mediated vasodilatation, as both of these oxygen radicals interact with each other (Mugge et al. 1991). However, Majid and Nishiyama (2002) observed that the renal vasoconstriction responses induced by DETC infusion were greatly enhanced in the absence of NO generation. The exact mechanism by which superoxide anions induce vasoconstriction is not clear but it has been reported that excessive intracellular calcium accumulates within myocardial tissue as a consequence of oxidative stress under ischemic conditions (Chakraborti et al. 1998). Thus, it is possible that an increase in intracellular calcium level induced by superoxide 
generation in vascular smooth muscle might induce a direct vasoconstriction.

The overall magnitude of the decrease in medullary perfusion in the SHRSP that we witnessed was comparable to that obtained in the Wistar rats which, at face value, may seem counterintuitive. However, we speculate that although SOD scavenges all or most metabolically produced superoxide anions under normal conditions (e.g. in Wistar rats), this may not be the case in the SHRSPs, where the reduced basal MBP (relative to Wistars) may be a reflection of increased levels of superoxide anions effectively 'swamping' SOD in these animals, thereby exerting the basal medullary vasoconstriction observed here. The possibility also exists that SHRSPs simply constitutively express less SOD than Wistar rats. However, we are unaware of any studies which strongly support such a suggestion for decreased SOD levels in these animals compared to normotensive controls. The only findings that partially support this hypothesis were originated from our previous study on the same animals, where we infused tempol, the compound that mimics the SOD, locally into $\mathrm{CMB}$, and observed a significant elevation in the MBP in SHRSP compared with Wistar rats (Ahmeda et al. 2017). These findings indicated that the supplementation of SOD system (by tempol), in the hypertensive rats, reduces the tone of the blood vessels and that might reflect the reduced level of SOD enzyme in the kidney region of SHRSPs.

The findings of the present study are comparable to those of previous investigations demonstrating that renal intra-arterial administration of DETC in anaesthetised dogs enhances superoxide anion activity in the kidney, resulting in increased renal vascular resistance and a reduction in the basal level of renal blood flow (Majid and Nishiyama 2002).

Our data suggest that the inhibition of SOD in SHRSPs allows the production of extra superoxide anions (relative to basal conditions) which might counteract the action of vasodilators such as NO, subsequently leading to further vasoconstriction and a reduction in medullary perfusion. This is supported by the findings suggesting that the presence of superoxide anions may diminish the half-life of NO (Haas et al. 1999, Beckman and Crow 1993). Furthermore, under normal conditions, endogenously produced superoxide anion in the renal medulla is largely scavenged by SOD and maintains a stable control of vascular resistance and vascular tone.

DETC infusion had no role in controlling BP when administered locally into the medulla for this short period. Moreover, DETC had no significant impact on cortical perfusion, which suggests that, assuming DETC was penetrating into the cortical tissue; the contribution of SOD and ROS in both hypertensive and normotensive animals to cortical perfusion was relatively minor. However, due to the fact that perfusion of the cortex is primarily controlled at the level of the glomerular arterioles, the possibility remains that DETC may not have reached the renal cortex at sufficient concentration to exert a significant effect on CBP in the superficial cortex, which necessitates diffusion of DETC across the outer medulla and a large part of the cortex.

\section{Conclusions}

Intra-medullary infusion of DETC exaggerated effect of oxidative stress by blocking the endogenous protective effect of SOD enzyme. This led to an accumulation of superoxide anions which, in turn, resulted in the constriction of medullary blood vessels and a consequent reduction in medullary blood perfusion. However, it should be noted that the magnitude of this vasoconstrictor effect was similar in both hypertensive and normotensive animals. Thus, we have confirmed that SOD is indeed an important regulator of MBP within both normal and hypertensive states.

\section{Conflict of Interest}

There is no conflict of interest.

\section{Acknowledgements}

The authors appreciate the "Research Centre of the Female Scientific and Medical Colleges", Deanship of Scientific Research at King Saud University for supporting this research.

\section{References}

AHMEDA AF, ALZOGHAIBI M: Factors regulating the renal circulation in spontaneously hypertensive rats. Saudi $J$ Biol Sci 23: 441-451, 2016. 
AHMEDA AF, JOHNS EJ: The regulation of blood perfusion in the renal cortex and medulla by reactive oxygen species and nitric oxide in the anaesthetised rat. Acta Physiol (Oxf) 204: 443-450, 2012.

AHMEDA AF, RAE MG, JOHNS EJ: Effect of reactive oxygen species and nitric oxide in the neural control of intrarenal haemodynamics in anaesthetized normotensive rats. Acta Physiol (Oxf) 209: 156-166, 2013.

AHMEDA AF, RAE MG, AL OTAIBI MF, ANWEIGI LM, JOHNS EJ: Effect of tempol and tempol plus catalase on intra-renal haemodynamics in spontaneously hypertensive stroke-prone (SHSP) and Wistar rats. $J$ Physiol Biochem 73: 207-214, 2017.

BECKMAN JS, CROW JP: Pathological implications of nitric oxide, superoxide and peroxynitrite formation. Biochem Soc Trans 21: 330-334, 1993.

BERECEK KH, SCHWERTSCHLAG U, GROSS F: Alterations in renal vascular resistance and reactivity in spontaneous hypertension of rats. Am J Physiol 238: H287-H293, 1980.

CHAKRABORTI T, GHOSH SK, MICHAEL JR, BATABYAL SK, CHAKRABORTI S: Targets of oxidative stress in cardiovascular system. Mol Cell Biochem 187: 1-10, 1998.

CHEN YF, COWLEY AW, ZOU AP: Increased $\mathrm{H}(2) \mathrm{O}(2)$ counteracts the vasodilator and natriuretic effects of superoxide dismutation by tempol in renal medulla. Am J Physiol Regul Integr Comp Physiol 285: R827-R833, 2003.

COWLEY AW, MATTSON DL, LU S, ROMAN RJ: The renal medulla and hypertension. Hypertension 25: 663-673, 1995.

HAAS JA, KRIER JD, BOLTERMAN RJ, JUNCOS LA, ROMERO JC: Low-dose angiotensin II increases free isoprostane levels in plasma. Hypertension 34: 983-986, 1999.

HALLIWELL B, GUTTERIDGE JM: Oxygen free radicals and iron in relation to biology and medicine: some problems and concepts. Arch Biochem Biophys 246: 501-514, 1986.

HARRISON DG: Endothelial function and oxidant stress. Clin Cardiol 20 (11 Suppl 2): II-11-7, 1997.

KERR S, BROSNAN MJ, MCINTYRE M, REID JL, DOMINICZAK AF, HAMILTON CA: Superoxide anion production is increased in a model of genetic hypertension: role of the endothelium. Hypertension 33: 1353-1358, 1999.

MAJID DS, NISHIYAMA A: Nitric oxide blockade enhances renal responses to superoxide dismutase inhibition in dogs. Hypertension 39: 293-297, 2002.

MORI T, OGAWA S, COWELY AW, ITO S: Role of renal medullary oxidative and/or carbonyl stress in salt-sensitive hypertension and diabetes. Clin Exp Pharmacol Physiol 39: 125-131, 2012.

MOSS NG, VOGEL PA, KOPPLE TE, ARENDSHORST WJ: Thromboxane-induced renal vasoconstriction is mediated by the ADP-ribosyl cyclase CD38 and superoxide anion. Am J Physiol Renal Physiol 305: F830-F838, 2013.

MUGGE A, ELWELL JH, PETERSON TE, HARRISON DG: Release of intact endothelium-derived relaxing factor depends on endothelial superoxide dismutase activity. Am J Physiol 260: C219-C225, 1991.

NAGAOKA A, KAKIHANA M, SUNO M, HAMAJO K: Renal hemodynamics and sodium excretion in stroke-prone spontaneously hypertensive rats. Am J Physiol 241: F244-F249, 1981.

OKAMOTO K, AOKI K: Development of a strain of spontaneously hypertensive rats. Jpn Circ J 27: 282-293, 1963.

OKAMOTO K, YAMORI Y, NAGAOKA A: Establishment of the stroke-prone spontaneously hypertensive rat (SHR). Circ Res 34: 143-153, 1974.

PARK JB, TOUYZ RM, CHEN X, SCHIFFRIN EL: Chronic treatment with a superoxide dismutase mimetic prevents vascular remodeling and progression of hypertension in salt-loaded stroke-prone spontaneously hypertensive rats. Am J Hypertens 15: 78-84, 2002.

STAICULESCU MC, FOOTE C, MEININGER GA, MARTINEZ-LEMUS LA: The role of reactive oxygen species in microvascular remodeling. Int J Mol Sci 15: 23792-23835, 2014.

SUZUKI H, SWEI A, ZWEIFACH BW, SCHMID-SCHONBEIN GW: In vivo evidence for microvascular oxidative stress in spontaneously hypertensive rats. Hydroethidine microfluorography. Hypertension 25: 1083-1089, 1995. 
TAGAMI M, NARA Y, KUBOTA A, SUNAGA T, MAEZAWA H, FUJINO H, YAMORI Y: Ultrastructural characteristics of occluded perforating arteries in stroke-prone spontaneously hypertensive rats. Stroke 18: 733-740, 1987.

VOLL RE, MIKULOWSKA A, KALDEN JR, HOLMDAHL R: Amelioration of type II collagen induced arthritis in rats by treatment with sodium diethyldithiocarbamate. J Rheumatol 26: 1352-1358, 1999.

ZOU AP, LI N, COWLEY AW: Production and actions of superoxide in the renal medulla. Hypertension 37: 547-553, 2001. 